\title{
Qualidade de Vida de Pacientes Oncológicos em Cuidados Paliativos Domiciliares e Desafios da Prática Médica diante da Finitude da Vida
}

doi: https://doi.org/10.32635/2176-9745.RBC.2020v66n4.423

\author{
Quality of Life of Cancer Patients in Home Palliative Care and Challenges of Medical Practice Facing the Finitude of Life \\ Calidad de Vida de los Pacientes con Cáncer en Cuidados Paliativos en el Hogar y Desafíos de la Práctica Médica que \\ Enfrentan la Finitud de la Vida
}

Vânia Nazaré Maia dos Santos'; Ana Cristina Soeiro²; Cristiane Ribeiro Maués ${ }^{3}$

\section{RESUMO}

Introduçáo: Os cuidados paliativos (CP) despontam como importante abordagem terapêutica diante do avanço do câncer. Objetivo: Identificar os fatores que interferem na qualidade de vida $(\mathrm{QV})$ de pacientes em CP domiciliares e discutir questôes relacionadas à prática médica no cuidado em saúde. Método: Pesquisa quanti-qualitativa, transversal e descritiva, com participação de nove pacientes em acompanhamento domiciliar no Hospital Ophir Loyola (HOL), e utilização do Palliative Outcome Scale (POS), do Questionário de Perfil Pessoal do Paciente e do Questionário de Qualidade de Vida. Resultados: Todos os participantes foram diagnosticados há mais de um ano e estavam em CP há mais de seis meses, sendo que a maioria já havia realizado diferentes modalidades terapêuticas, destacando-se a quimioterapia. O principal local de tumor primário foi a mama e, entre os sintomas físicos apresentados, a presença de dor foi relatada pela maioria dos participantes. Categorias analíticas emergiram do tema QV, sendo a manutenção da saúde, o convívio familiar, o equilíbrio financeiro, a importância da fé e a esperança, a realizaçáo de atividades cotidianas e laborais e a manutenção da autonomia identificadas como variáveis que interferem positivamente na QV. As dificuldades socioeconômicas foram associadas a piores desempenhos no POS. Todos apresentaram uma avaliação positiva do trabalho desenvolvido pela equipe de CP. Conclusáo: Agravos clínicos e sociais, com destaque para a dor e as limitaçôes econômicas, são fatores que interferem na QV dos pacientes em atendimento domiciliar. Apesar dos avanços alcançados, a medicina ainda necessita priorizar a terminalidade da vida como parte indissociável da formação e prática médica. Palavras-chave: Neoplasias; Cuidados Paliativos; Assistência Domiciliar; Qualidade de vida.

\section{ABSTRACT}

Introduction: Palliative Care (PC) emerges as an important therapeutic approach in face of cancer progress. Objective: To identify the factors that affect the quality of life $(\mathrm{QoL})$ of patients in home $\mathrm{PC}$ and discuss issues related to medical practice in health care. Method: Quantitative, qualitative, cross-sectional and descriptive study, involving 9 patients in home care at Ophir Loyola Hospital (HOL), using the Palliative Outcome Scale (POS), Patient Personal Profile Questionnaire and Quality of Life Questionnaire. Results: All the participants were diagnosed for over a year and had been in palliative care for more than six months, and most have already submitted to different therapeutic modalities, especially chemotherapy. Breast was the main site of primary tumor and among the physical symptoms presented, the presence of pain was reported by the majority of the participants. Analytical categories emerged from the theme quality of life, and the maintenance of health, family life, financial balance, importance of faith and hope, performance of daily and work activities and maintenance of autonomy were identified as variables that positively affect QoL. Socioeconomic difficulties were associated with worse performances in POS. All presented positive evaluation of the work done by the PC team. Conclusion: Clinical and social problems, especially pain and economic limitations are factors that interfere with the QoL of patients in home care. Despite the advances achieved, medicine still needs to prioritize the terminality of life as an inseparable part of medical education and practice.

Key words: Neoplasms; Palliative Care; Home Nursing; Quality of life.

\section{RESUMEN}

Introducción: Los cuidados paliativos (PC) emergen como un enfoque terapéutico importante frente al progreso del cáncer. Objetivo: Identificar los factores que afectan la calidad de vida (QOL) de los pacientes con PC en el hogar y discutir temas relacionados con la práctica médica en la atención médica. Método: Estudio cuantitativo, cualitativo, transversal y descriptivo, que involucró a nueve pacientes en atención domiciliaria en el Hospital Ophir Loyola (HOL), utilizando el Palliative Outcome Scale (POS), el Cuestionario de perfil personal del paciente y el Cuestionario de Calidad de Vida. Resultados: Todos los participantes fueron diagnosticados durante más de un año y habían estado en PC durante más de seis meses, y la mayoría ya había realizado diferentes modalidades terapéuticas, especialmente quimioterapia. El sitio principal del tumor primario fue la mama y entre los síntomas físicos presentados, la mayoría de los participantes informaron la presencia de dolor. Las categorías analíticas surgieron del tema calidad de vida, y el mantenimiento de la salud, la vida familiar, el equilibrio financiero, la importancia de la fe y la esperanza, el desempeńo de las actividades diarias y laborales y el mantenimiento de la autonomía se identificaron como variables que afectan positivamente la calidad de vida. Las dificultades socioeconómicas se asociaron con peores desempeños en POS. Todos tuvieron una evaluación positiva del trabajo realizado por el equipo de PC. Conclusión: Los problemas clínicos y sociales, especialmente el dolor y las limitaciones económicas, son factores que interfieren con la QOL de los pacientes en atención domiciliaria. A pesar de los avances logrados, la medicina aún necesita priorizar la terminación de la vida como una parte inseparable de la educación y práctica médica.

Palabra clave: Neoplasias; Cuidados Paliativos; Atención Domiciliaria de Salud; Calidade de Vida.

'Universidade do Estado do Pará (UEPA). Belém (PA), Brasil. Orcid iD: https://orcid.org/0000-0002-1121-4843

2UEPA. Belém (PA), Brasil. Orcid iD: https://orcid.org/0000-0002-1669-3839

${ }^{3}$ Centro Universitário do Pará. UEPA. Hospital Ophir Loyola. Belém (PA), Brasil. Orcid iD: https://orcid.org/0000-0002-2338-7944

Endereço para correspondência: Vânia Nazaré Maia dos Santos. Avenida Conselheiro furtado, 1640 - Batista Campos. Belém (PA), Brasil. CEP 66063-060. E-mail: vaniamaiasantos@gmail.com 


\section{INTRODUÇÃO}

Nas últimas décadas, o câncer tem alcançado patamares alarmantes, sendo considerado um grave problema de saúde pública e uma das principais causas de morte na população mundial ${ }^{1,2}$. Estimativas apontam que, para o Brasil, no triênio 2020-2022, ocorrerão a cada ano ${ }^{3}$ cerca de 625 mil casos novos de câncer, o que revela a magnitude do problema também no contexto nacional ${ }^{4}$.

Como princípios fundamentais dos cuidados paliativos (CP), é enfatizada a prevenção e o alívio do sofrimento, a identificação precoce, a avaliação impecável e o tratamento de dor e demais sintomas físicos, sociais, psicológicos e espirituais ${ }^{5}$. Embora fortemente relacionados à oncologia, os CP têm se expandido para outras áreas da prática médica, sendo recomendados desde o momento em que o paciente recebe o diagnóstico, se estendendo até mesmo após a sua morte, em apoio ao luto da família ${ }^{6}$.

Diante do aumento de novos casos e da própria evolução da doença, a temática dos CP tem despontado como uma importante filosofia do cuidado em saúde, em especial quando as chances de cura se revelam improváveis, mesmo frente aos grandes avanços terapêuticos dos últimos anos ${ }^{6,7}$. Partindo do reconhecimento da morte como evento natural, os CP defendem a abordagem ativa e integral, promovida por uma equipe multidisciplinar, que objetiva a melhoria da qualidade de vida do paciente (QV) e de seus familiares diante de uma doença que ameace a vida ${ }^{7,8}$.

Os CP estão intrinsicamente relacionados à busca por melhor QV dos pacientes que enfrentam doenças graves e incuráveis, o que representa um desafio nas práticas em saúde, especialmente pela íntima relação com o tema da terminalidade da vida ${ }^{6-8}$. Nos dias atuais, e mesmo na medicina, as questôes ligadas à morte e ao morrer ainda permanecem sendo uma área obscura e problemática, o que se reflete no pouco preparo para a atuação em $\mathrm{CP}^{9}$.

Vale lembrar, conforme definição proposta pela Organização Mundial da Saúde (OMS), que a QV inclui a percepção do indivíduo em relação às influências sociais, culturais, políticas e econômicas no contexto de sua vida, para o alcance de seus objetivos, projetos e expectativas, conferindo-lhe oportunidades de escolhas; ou seja, reflete sua autonomia e sua satisfação com a vida ${ }^{10}$.

Em 2018, no Brasil, os CP foram instituídos como política pública e reconhecidos como parte dos cuidados continuados integrados no âmbito do Sistema Único de Saúde (SUS), podendo ser realizados em diversos cenários, incluindo enfermarias hospitalares, instituições de longa permanência, ambulatórios especializados e em domicílio $^{11-13}$.

A promoção de $\mathrm{CP}$ em ambiente domiciliar permite aos pacientes a possibilidade de permanecer em seu contexto familiar e social ${ }^{14,15}$, com atenção multiprofissional, especializada e disposta a oferecer suporte e orientação aos familiares e/ou cuidadores, evitando internaçôes recorrentes e muitas vezes desnecessárias ${ }^{16,17}$. Entretanto, os cuidados domiciliares ainda são uma prática pouco frequente em muitos locais, haja vista a sua forte predominância nos espaços hospitalares ${ }^{17}$.

Apesar do crescente interesse pelo tema, a realização de pesquisas sobre QV em pacientes que se encontram em CP oncológico é fundamental para identificar os domínios afetados e assim planejar as intervenções a serem realizadas ${ }^{18-21}$. Estudos dessa natureza tem o potencial para contribuir para a efetivação das políticas públicas de saúde, orientando programas e condutas terapêuticas, possibilitando, inclusive, uma melhor difusão do ensino de CP nas escolas médicas brasileiras ${ }^{22-24}$

Considerando a importância da temática e a escassez de produção científica sobre o assunto, a pesquisa foi planejada com o objetivo de identificar os fatores que interferem na QV de pacientes em CP domiciliares, atendidos em um hospital público oncológico no Pará. Trata-se de um tema complexo, mas necessário de ser problematizado, considerando as questóes envolvidas; em especial, aquelas relacionadas ao cuidado diante da finitude humana e suas implicaçóes para a prática médica.

\section{MÉTODO}

Pesquisa com abordagem quanti-qualitativa, transversal e descritiva, realizada nas residências dos pacientes atendidos em CP domiciliar pelo Hospital Ophir Loyola (HOL). O hospital funciona como referência em oncologia e CP no Pará e foi o primeiro a inaugurar uma Clínica de Cuidados Paliativos Oncológicos (CCPO) em nível estadual. Ao todo, foram incluídos nove pacientes, os quais se encontravam em acompanhamento sistemático pela CCPO no período da coleta de dados, e que aceitaram participar voluntariamente da pesquisa.

No protocolo da pesquisa, foram utilizados três instrumentos, incluindo o Palliative Outcome Scale (POS), validado nacionalmente e composto por 12 itens, incorporando aspectos sobre os sintomas de dor, sintomas em geral, bem-estar psicológico, ansiedade da família, qualidade dos CP e dificuldades pessoais e financeiras, de acordo com reaçóes apresentadas nos últimos três dias. Também foi utilizado o Questionário de Perfil Pessoal do Paciente, elaborado pelos pesquisadores, que incluiu dados sociodemográficos e perguntas sobre o perfil clínico, com informaçóes sobre o tipo de doença, tempo de diagnóstico, tratamentos já realizados e tempo de ingresso nos CP. O terceiro instrumento foi o Questionário de Qualidade de Vida, contendo três perguntas abertas: 1) "Na sua opinião, 
o que é qualidade de vida?"; 2) "Na sua opiniáo, quais os fatores que auxiliam e dificultam a sua qualidade de vida?"; 3) "De que modo o trabalho prestado pela equipe de saúde da CCPO interfere em sua qualidade de vida?".

A coleta de dados foi realizada por meio de entrevistas aos pacientes cadastrados na visita domiciliar mediante seu consentimento prévio e disponibilidade, sem prejuízo para a assistência. As entrevistas foram gravadas com auxílio de gravador e transcritas na íntegra, sendo o número de participantes definido em conjunto com a equipe de saúde e conforme a demanda clínica.

Os dados foram interpretados por meio de análise quantitativa, com base na estatística descritiva, com uso dos programas Excel e Statisticaal Package for the Social Science (SPSS). Em relação às perguntas abertas, a análise de dados foi feita com uso de categorias descritivas, as quais foram organizadas previamente segundo os objetivos propostos. As repostas foram posteriormente apresentadas segundo as categorias temáticas extraídas a partir dos dados obtidos.

A presente pesquisa foi aprovada pelo Comitê de Ética em Pesquisa (CEP) da Universidade do Estado do Pará (UEPA) e do HOL, sob o número de parecer: 3.174.496. Antes da realização das entrevistas, foi disponibilizado aos participantes o Termo de Consentimento Livre e Esclarecido (TCLE) em duas vias de igual teor; uma cópia permaneceu com o participante da pesquisa e a outra com os pesquisadores. Para manter o anonimato, os participantes foram identificados com uso de código alfanumérico.

\section{RESULTADOS}

A maioria dos pacientes incluídos no estudo era do sexo feminino $(n=7,77,7 \%)$, negros $(n=4,77,7 \%)$, divorciados $(n=4,44,4 \%)$ e de religião evangélica $(n=5$, $55,5 \%$ ). Parte majoritária se situava na faixa etária acima de 80 anos $(n=4,44,4 \%)$, seguida de 60 a 80 anos $(\mathrm{n}=3,33,3 \%)$, e dois pacientes tinham entre 30 a 59 anos $(22,3 \%)$. Cabe destacar que a amostra apresentava consideráveis dificuldades socioeconômicas: quatro pacientes $(44,4 \%)$ tinham renda familiar de apenas um salário mínimo, três pacientes $(33,3 \%)$ tinham renda de um a dois salários e apenas dois tinham renda acima de dois salários mínimos. Em relação à procedência, seis pacientes $(66,65 \%)$ procediam da capital e três $(33,35 \%)$, do interior (Tabela 1).

Os resultados obtidos com o POS apontaram um escore médio maior, portanto, mais desfavorável nos pacientes com renda familiar inferior a um salário mínimo (média de 20 pontos), em comparaçáo com os que tinham de um a dois salários (média de 13,3 pontos) e acima de
Tabela 1. Distribuição de pacientes segundo características sociodemográficas - frequências absolutas e relativas. Belém-PA, 2019

\begin{tabular}{|c|c|c|c|}
\hline $\begin{array}{c}\text { Características } \\
\text { sociodemográficas }\end{array}$ & Categorias & $\mathbf{N}$ & $\%$ \\
\hline \multirow{3}{*}{ Sexo } & Feminino & 7 & 77,7 \\
\hline & Masculino & 2 & 22,3 \\
\hline & Preto & 4 & 44,4 \\
\hline \multirow[t]{3}{*}{ Raça } & Pardo & 3 & 33,3 \\
\hline & Branco & 2 & 22,3 \\
\hline & Casado & 3 & 33,3 \\
\hline \multirow{4}{*}{ Estado civil } & Divorciado & 4 & 44,4 \\
\hline & União estável & 1 & 1,15 \\
\hline & Viúvo & 1 & 1,15 \\
\hline & $30-59$ anos & 2 & 22,3 \\
\hline \multirow[t]{2}{*}{ Faixa etária } & $60-80$ anos & 3 & 33,3 \\
\hline & $>80$ anos & 4 & 44,4 \\
\hline \multirow{2}{*}{ Religião } & Evangélico & 5 & 55,5 \\
\hline & Católico & 4 & 44,4 \\
\hline \multirow{3}{*}{ Faixa de renda } & $<1$ salário & 4 & 44,4 \\
\hline & 1-2 salários & 3 & 33,3 \\
\hline & >2 salários & 2 & 22,3 \\
\hline \multirow{2}{*}{ Residência } & Capital & 6 & 66,6 \\
\hline & Interior & 3 & 33,3 \\
\hline
\end{tabular}

Fonte: Protocolo de pesquisa.

dois salários (média 9). Do mesmo modo, também foi constatado um escore médio do POS pior nos pacientes divorciados (média de 17,25), em relação aos casados (média de 16,6 pontos) e com uniáo estável (15 pontos).

$\mathrm{Na}$ Tabela 2, são apresentadas as características clinicopatológicas. Considerando as propriedades mais relevantes, os principais locais de tumores primário foram mama $(n=5,55,5 \%)$, reto $(n=2,22,2 \%)$, próstata e orofaringe, ambos com $\mathrm{n}=1$ (11,15\% cada). Todos os pacientes da amostra $(n=9)$ haviam sido diagnosticados há mais de 1 ano. Em relaçẫo aos tratamentos feitos anteriormente, houve realização prévia de quimioterapia $\mathrm{n}=1 \quad(11,1 \%)$, quimioterapia associada à cirurgia $\mathrm{n}=2(22,25 \%)$, quimioterapia associada à cirurgia e à radioterapia $n=3(33,35 \%)$, cirurgia associada à radioterapia $\mathrm{n}=1(11,1 \%)$, quimioterapia associada à radioterapia $\mathrm{n}=1(11,1 \%)$ e radioterapia isolada $\mathrm{n}=1$ $(11,1 \%)$.

Considerando as respostas aos itens do POS, pode-se perceber que, em relação à dor, oito pacientes $(88,8 \%)$ relataram sentir dor, sendo $33 \%$ de intensidade leve, $44,4 \%$ moderada e $11,1 \%$ grave. Em relaçáo à presença de outros sintomas físicos desconfortáveis, $77,7 \% \mathrm{da}$ amostra afirmou apresentar algum tipo de sintoma, com 
Tabela 2. Distribuição de pacientes segundo características clinicopatológicas - frequências absolutas e relativas. Belém-PA, 2019

\begin{tabular}{|c|c|c|c|}
\hline $\begin{array}{l}\text { Características } \\
\text { clinicopatológicas }\end{array}$ & Categorias & $\mathbf{N}$ & $\%$ \\
\hline \multirow{4}{*}{ Tipo de câncer } & Câncer de mama & 5 & 55,5 \\
\hline & $\begin{array}{l}\text { Câncer de } \\
\text { próstata }\end{array}$ & 1 & 11,1 \\
\hline & Câncer de reto & 2 & 22,2 \\
\hline & $\begin{array}{l}\text { Câncer de } \\
\text { orofaringe }\end{array}$ & 1 & 11,1 \\
\hline \multirow{4}{*}{$\begin{array}{l}\text { Tempo de } \\
\text { diagnóstico }\end{array}$} & Menos de 1 ano & 0 & \\
\hline & Mais de 1 ano & 9 & 100 \\
\hline & QT & 1 & 11,1 \\
\hline & QT + cirurgia & 2 & 22,2 \\
\hline \multirow[t]{5}{*}{ Tratamento } & $\begin{array}{l}\text { QT+ cirurgia + } \\
\text { RxT }\end{array}$ & 3 & 33,3 \\
\hline & Cirurgia + RxT & 1 & 11,1 \\
\hline & $\mathrm{QT}+\mathrm{RxT}$ & 1 & 11,1 \\
\hline & $\mathrm{RxT}$ & 1 & 11,1 \\
\hline & 1-3 meses & 3 & 33,3 \\
\hline \multirow{3}{*}{$\begin{array}{l}\text { Tempo nos cuidados } \\
\text { paliativos }\end{array}$} & 3-6 meses & 1 & 11,1 \\
\hline & 6 meses- 1 ano & 2 & 22,2 \\
\hline & Acima de 1 ano & 3 & 33,3 \\
\hline
\end{tabular}

Fonte: Prontuários da Clínica de Cuidados Paliativos Domiciliares do Hospital Ophir Loyola.

Legendas: $\mathrm{QT}=\mathrm{Quimioterapia;} \mathrm{Rx}=$ Radioterapia.

intensidade variando de moderada (44,4\%), leve $(22,2 \%)$ e grave $(11,1 \%)$.

Pode-se constatar que parte majoritária da amostra $(55,5 \%)$ referiu ansiedade ou preocupações em relação à sua doença/tratamento. Em se tratando da percepção do paciente sobre a reaçáo de familiares diante da doença, $33,3 \%$ perceberam a preocupação dos familiares na maior parte do tempo, 22,2\% tiveram a percepçáo algumas vezes e $11,1 \%$, pouquíssimas vezes. Entretanto, cinco pacientes $(55,5 \%)$ referiram dificuldades para expressar e compartilhar seus sentimentos com familiares.

Em se tratando das informaçóes recebidas pelo paciente sobre a doença, $55,55 \%$ da amostra referiram ter recebido as informações necessárias e 44,45\% relataram ter recebido também informaçôes, ressaltando, porém, que tiveram dificuldade em compreendê-las.

Em relaçáo aos itens que refletem o bem-estar psicológico dos pacientes, quase todos os pacientes acreditam que sua vida vale a pena, alterando-se a ocorrência desse sentimento: "o tempo todo" ( $\mathrm{n}=4,44,4$ $\%)$, "na maior parte das vezes" ( $\mathrm{n}=2,22,2 \%)$, "algumas vezes" ( $\mathrm{n}=2,22,2 \%)$ e "não, nem um pouco" ( $\mathrm{n}=1,11,1$ $\%)$. Constatou-se ainda que dois pacientes (22,2\%) se sentiram bem consigo mesmos "o tempo todo", três pacientes $(33,3 \%)$ sentiram-se assim "na maior parte do tempo", três pacientes (33,3\%) sentiram-se assim "algumas vezes", um paciente sentiu-se bem consigo mesmo "pouquíssimas vezes" (11,1\% da amostra).

Verificou-se que $55,5 \%$ dos pacientes dispendem até metade de um dia com compromissos relacionados à sua saúde, incluindo atividades de autocuidado e outras relacionadas às prescriçôes terapêuticas.

Os problemas financeiros relacionados ao processo de adoecimento despontaram como uma das principais dificuldades enfrentadas por seis participantes $(66,6 \%)$, com destaque para os gastos com a compra medicamentos e fraldas, conforme exemplificado em dois depoimentos: "meu marido parou de trabalhar para cuidar de mim", "falta dinheiro para comida". Além disso, quatro pacientes $(44,4 \%)$ referiram problemas pessoais, tal como "perda da autonomia e maior dependência".

Os dados obtidos por meio da entrevista com perguntas abertas sobre o significado de QV para o paciente, os fatores de melhora e de piora dessa QV e o papel da equipe de saúde do CP domiciliar na QV estấo contidos nos Quadros 1, 2 e 3.

\section{DISCUSSÃO}

A maioria dos participantes do estudo se encontrava na faixa etária acima dos 60 anos, corroborando outras pesquisas em níveis nacional e regional, fato possivelmente relacionado à grande incidência de doenças crônico-degenerativas nessa parcela da população $0^{3,25-28}$. O avanço da idade é um dos fatores que pode influenciar o prognóstico da doença e a necessidade de CP oncológicos ${ }^{12,19}$, em especial quando há atraso no diagnóstico e os pacientes já ingressam no tratamento sem chances de cura da doença, uma realidade inclusive bastante frequente no contexto em questão (informação verbal).

Do total de participantes, a maioria era do sexo feminino $(77,7 \%)$, resultado também semelhante ao encontrado na literatura ${ }^{9,24,26}$. Em países em desenvolvimento, como o Brasil, é esperado o predomínio de câncer no sexo feminino, em virtude dos altos índices de neoplasias na população feminina, a exemplo dos cânceres do colo uterino e de mama, de grande prevalência no Estado. Para cada ano do triênio de 2020-2022, são esperados 66 mil casos de câncer de mama ${ }^{3,29}$. Em contrapartida, em países desenvolvidos, a ocorrência de câncer tende a incidir de forma similar em ambos os sexos, por causa dos programas de prevenção em massa desenvolvidos por esses países ${ }^{14,18}$.

Os participantes incluídos no estudo eram de maioria negra (pretos $44,4 \%$ e pardos $33,3 \%$ ), o que pode ser explicado pelo fato de $70 \%$ dos usuários atendidos no 
Quadro 1. Distribuição de categorias e conteúdo das respostas obtidas sobre compreensão sobre a expressão "qualidade de vida" na entrevista com pacientes em cuidado paliativo domiciliar. Belém-PA, 2019

\begin{tabular}{|c|c|c|}
\hline $\begin{array}{l}\text { Categorias } \\
\text { analíticas }\end{array}$ & $\begin{array}{l}\text { Categorias } \\
\text { empíricas }\end{array}$ & $\begin{array}{l}\text { Trecho dos } \\
\text { depoimentos }\end{array}$ \\
\hline $\begin{array}{l}\text { Compreensão } \\
\text { sobre a } \\
\text { expressão } \\
\text { "qualidade de } \\
\text { vida" }\end{array}$ & $\begin{array}{l}\text { Realização } \\
\text { de atividades } \\
\text { cotidianas e } \\
\text { laborais } \\
\text { Manutenção } \\
\text { da } \\
\text { autonomia }\end{array}$ & $\begin{array}{l}\text { "É ter saúde" } \\
\text { (P9) } \\
\text { "Estar com a } \\
\text { família" (P1) } \\
\text { "É uma vida } \\
\text { onde a pessoa } \\
\text { possa comprar } \\
\text { tudo que } \\
\text { precisa, né? } \\
\text { Alimentação } \\
\text { principalmente" } \\
\text { (P3) } \\
\text { "Ficar bom pra } \\
\text { voltar a fazer } \\
\text { o que eu fazia } \\
\text { (trabalhar)" (P4) } \\
\text { "É porque a } \\
\text { gente com } \\
\text { saúde pode } \\
\text { trabalhar" (P9) } \\
\text { "É ter minha } \\
\text { saúde, andar } \\
\text { como eu } \\
\text { andava. Agora } \\
\text { eu não posso } \\
\text { fazer meu café, } \\
\text { dependo dos } \\
\text { outros pra tudo" } \\
\text { (P8) }\end{array}$ \\
\hline
\end{tabular}

Fonte: Protocolo de pesquisa.

SUS serem negros ${ }^{30}$. Na perspectiva socioeconômica, a grande maioria dos pacientes da amostra tinha renda familiar menor ou igual a dois salários mínimos, sendo que $44,4 \%$ tinham renda menor ou igual a um salário, o que pode estar correlacionado ao perfil da população atendida pelo hospital ${ }^{26}$. Embora se trate de uma instituição pública de referência no tratamento oncológico, com a recente criação de diversas clínicas e unidades hospitalares, houve a migração de muitos pacientes particulares para esses espaços, o que, em parte, explica a forte de presença de pessoas oriundas das classes populares sendo atendidas nesse cenário. Tais achados merecem relevância, visto que as iniquidades sociais interferem na $\mathrm{QV}^{31-35}$ e a mortalidade, em pacientes oncológicos ${ }^{32,33}$.
Quadro 2. Distribuição de categorias e conteúdo das respostas obtidas sobre fatores que influenciam a qualidade de vida na entrevista com pacientes em cuidado paliativo domiciliar. Belém-PA, 2019

\begin{tabular}{|c|c|c|}
\hline $\begin{array}{c}\text { Categorias } \\
\text { analíticas }\end{array}$ & $\begin{array}{l}\text { Categorias } \\
\text { empíricas }\end{array}$ & $\begin{array}{l}\text { Trecho dos } \\
\text { depoimentos }\end{array}$ \\
\hline \multirow[t]{4}{*}{$\begin{array}{l}\text { Fatores que } \\
\text { influenciam } \\
\text { qualidade de } \\
\text { vida }\end{array}$} & $\begin{array}{l}\text { Apoio familiar } \\
\text { e suporte } \\
\text { social }\end{array}$ & $\begin{array}{l}\text { "A fé me ajuda } \\
\text { muito, me alimenta } \\
\text { muito" (P1) } \\
\text { "Ter fé" (P2) } \\
\text { "Minha qualidade } \\
\text { de vida primeiro é } \\
\text { o bem-estar com } \\
\text { a própria família, } \\
\text { conviver bem" (P2) } \\
\text { "Apoio da família" } \\
\text { (P6, P8) } \\
\text { "O que melhora } \\
\text { são meus filhos } \\
\text { perto de mim, } \\
\text { não gosto de ficar } \\
\text { sozinha nenhum } \\
\text { instante" (P7) } \\
\text { "O que melhora é } \\
\text { quando tenho visita } \\
\text { dos meus parentes" } \\
\text { (P4) }\end{array}$ \\
\hline & $\begin{array}{l}\text { Condição } \\
\text { financeira }\end{array}$ & $\begin{array}{l}\text { "Ajuda a ter } \\
\text { qualidade de } \\
\text { vida, ter uma vida } \\
\text { financeira boa" (P3) } \\
\text { "O que piora é ter } \\
\text { preocupação com } \\
\text { dívida" (P2) } \\
\text { "O que piora é } \\
\text { quando falta as } \\
\text { coisas em casa, } \\
\text { quando falta } \\
\text { comida" (P9) }\end{array}$ \\
\hline & $\begin{array}{l}\text { Falta de } \\
\text { autonomia }\end{array}$ & $\begin{array}{l}\text { "O que atrapalha } \\
\text { é depender dos } \\
\text { outros" (P8) }\end{array}$ \\
\hline & $\begin{array}{l}\text { Presença de } \\
\text { dor }\end{array}$ & $\begin{array}{l}\text { "O que atrapalha } \\
\text { são as dores e ter } \\
\text { que abrir a boca } \\
\text { pra comer" (P4) }\end{array}$ \\
\hline
\end{tabular}

Fonte: Protocolo de pesquisa.

Vale destacar que o escore médio obtido no POS foi pior nos pacientes com renda familiar inferior a um salário mínimo, em comparaçáo ao restante da amostra. A relevância desse achado traz importantes 
Quadro 3. Distribuição de categorias e conteúdo das respostas obtidas sobre a influência da equipe de cuidados paliativos domiciliares na qualidade de vida na entrevista com pacientes em cuidado paliativo domiciliar. Belém-PA, 2019

\begin{tabular}{|c|c|c|}
\hline $\begin{array}{l}\text { Categorias } \\
\text { analíticas }\end{array}$ & $\begin{array}{l}\text { Categorias } \\
\text { empíricas }\end{array}$ & $\begin{array}{c}\text { Trecho dos } \\
\text { depoimentos }\end{array}$ \\
\hline $\begin{array}{l}\text { A influência } \\
\text { da equipe } \\
\text { de cuidados } \\
\text { paliativos } \\
\text { domiciliares na } \\
\text { qualidade de } \\
\text { vida }\end{array}$ & $\begin{array}{l}\text { Acesso a } \\
\text { diferentes } \\
\text { profissionais }\end{array}$ & $\begin{array}{l}\text { "Depois que elas } \\
\text { vieram aqui, } \\
\text { eu passei a ter } \\
\text { acompanhamento } \\
\text { de coisas que } \\
\text { nunca tive no } \\
\text { hospital, tipo } \\
\text { terapeuta, } \\
\text { fonoaudióloga, } \\
\text { dentista" (P4) } \\
\text { "Ajuda muito, sou } \\
\text { muito bem tratada } \\
\text { e muito bem } \\
\text { cuidada" (P1) } \\
\text { "Ah esse trabalho } \\
\text { é excepcional! } \\
\text { Ajuda, } \\
\text { ajuda, ajuda! } \\
\text { Principalmente } \\
\text { no Natal, eu } \\
\text { não esperava, } \\
\text { veio aqui uma } \\
\text { comissão, me } \\
\text { trouxeram uns } \\
\text { presentes aqui, } \\
\text { cesta básica" (P2) } \\
\text { "Eu gosto muito de } \\
\text { ver elas fazerem } \\
\text { isso comigo. Eu me } \\
\text { sinto bem" (P8, P9) }\end{array}$ \\
\hline
\end{tabular}

Fonte: Protocolo de pesquisa.

desdobramentos, uma vez que quase todos os pacientes referiram interferência econômica na sua QV. Quando indagados sobre sua percepção pessoal acerca de sua QV, o equilíbrio financeiro foi mencionado como um aspecto importante afetado pelo adoecimento, o que pode ser justificado pelos elevados custos com a manutençáo das condiçôes de saúde desses pacientes.

Quanto à procedência, observou-se que 66,6\% eram provenientes da capital e o restante, de outros municípios do Estado, fato que reflete o potencial impacto que o tratamento impóe na rotina de vida. Um dos fatores a serem considerados reside no fato de que muitos pacientes precisam se deslocar de seu local de origem para tratamento na região metropolitana ${ }^{25}$. Como as distâncias territoriais são longas e há muitas localidades de difícil acesso, há uma série de mudanças em decorrência do tratamento, as quais também podem demandar elevados custos ao paciente e à sua famíliaa ${ }^{25,31}$.

Em relação ao estado civil, 55,5\% dos pacientes pesquisados eram casados ou viviam em uniáo estável e $44,4 \%$ eram divorciados. Embora achados na literatura nacional não demonstrem claramente a importância do estado civil na QV de pacientes em CP, pacientes com câncer e que vivem em união estável ou casados têm mais chances de sobrevivência do que aqueles que vivem só $^{26,36,37}$. No presente estudo, os pacientes divorciados obtiveram os piores escores médios do POS $(17,25)$, em comparação aos casados $(16,6)$ e com união estável $(15)$, o que fornece indícios sobre a provável importância do suporte social prestado por pessoas próximas ao paciente no enfrentamento da doença.

Estudos sobre saúde e espiritualidade têm demonstrado a importância da fé e da religiosidade no enfrentamento do câncer, as quais estão associadas a menores níveis de ansiedade e depressão $0^{8,37,38}$. No que tange à religião, a maioria dos participantes era evangélica $(55,5 \%)$, seguida de católicos $(44,4 \%)$, que representam também os segmentos religiosos característicos da população atendida pelo hospital ${ }^{26}$.

A fé configurou-se como um dos fatores que contribuem para melhor a QV dos pacientes. Nesse sentido, muitos pacientes em CP procuram a espiritualidade como baluarte de sustentaçáo que proporciona melhor bem-estar ${ }^{6}$. As relaçôes entre espiritualidade e CP têm sido cada vez mais investigadas, apontando para uma relação, majoritariamente, positiva, contribuindo até em aspectos de ansiedade e depressão durante o processo patológico ${ }^{23,38}$.

No tocante aos aspectos clínicos dos pacientes com câncer em CP, destacam-se os dados apresentados pela Tabela 2. O câncer de mama obteve maior incidência (55,5\% da amostra), o que condiz com os achados de que essa é a segunda neoplasia maligna que mais acomete mulheres no Brasil, atrás apenas do câncer de pele não melanoma ${ }^{3,39}$. O segundo câncer mais incidente na referida amostra foi o de reto. Vale ressaltar que, dos tumores de pele náo melanoma, o câncer de cólon e reto em homens é o quarto mais incidente na Região Norte $(5,27 / 100 \text { mil })^{3}$.

Todos os pacientes tiveram o diagnóstico de câncer há mais de um ano, sendo que a maior parte $(55,5 \%)$ já estava nos CP há mais de seis meses. Cabe destacar que o controle do câncer envolve a promoçáo à saúde, reabilitaçáo e $\mathrm{CP}^{13,14,20,32}$, sendo este último regulamentado pelo Ministério da Saúde do Brasil como parte dos cuidados continuados integrados no âmbito do SUS pela Resolução n. ${ }^{\circ} 41$, de 31 de outubro de $2018^{11}$.

A progressão da doença neoplásica impóe ao paciente uma diversidade de sinais e sintomas que afetam de forma 
significativa a sua QV. Sob esse aspecto, a maioria $(88,8 \%)$ dos participantes deste estudo relatou a ocorrência de dor nos últimos três dias e 77,7\% referiram também presença de outros sintomas físicos desconfortáveis. Tais achados corroboram um estudo realizado em João Pessoa, no qual $89,8 \%$ da amostra referiram dor $^{7}$.

$\mathrm{Na}$ América Latina, o Brasil ocupa o segundo lugar entre os países onde pessoas com câncer relatam sentir mais o sintoma da dor, sendo que $24 \%$ a $46 \%$ dos pacientes com dor oncológica têm sua dor controlada de forma inadequada ${ }^{40,41}$.

A compreensão de que a dor suscita intenso desconforto físico e psicológico impóe à equipe que cuida de pacientes oncológicos e, principalmente, em fase avançada da doença, a necessidade do alívio do sintoma, o que representa um dos princípios estruturantes dos $\mathrm{CP}^{5}$. Quando não identificada e manejada, a dor potencializa os sintomas físicos, gerando não só desconforto físico, mas também elevando o sofrimento e interferindo diretamente na $\mathrm{QV}^{5,41}$.

Não foi possível correlacionar os achados de modo a inferir que a presença de dor poderia estar ligada à presença dos sintomas de ansiedade (55,5\%) relatados pelos participantes, mas, possivelmente, pelo menos em parte, talvez justifique porque alguns dos participantes relataram frequentes preocupaçóes acerca de sua doença/ tratamento.

Embora a maioria (55,5\%) da amostra tenha recebido informaçôes necessárias sobre a doença, cabe destacar que $44,45 \%$ relataram dificuldade em compreendê-las. Tais achados reforçam a importância de que a equipe de saúde esteja atenta às necessidades do paciente, incluindo informaçôes e explicaçōes claras e de fácil compreensão. Quando se considera o perfil socioeconômico dos pacientes atendidos pelo hospital, é de se esperar que muitos tenham dificuldade em entender as informações, o que é um requisito indispensável ao cuidado integral ${ }^{10,11,14,20}$, em especial quando o objetivo são os $\mathrm{CP}$.

A despeito do quadro clínico, observou-se que a maioria dos participantes valoriza a vida $(88,8 \%)$ e todos referiram sentir-se bem consigo mesmos, porém com frequências diferentes. Os achados indicam que a fé e a espiritualidade, bem como o apoio e suporte familiar, também são fatores que contribuem para a QV.

Dados presentes na literatura têm mostrado que os familiares constituem o pilar de apoio social e emocional para pacientes, pois desempenham papel importante no processo saúde-doença ${ }^{6,41}$. Estudos anteriores avaliaram a QV de pacientes com câncer em diferentes estágios de evolução e demonstraram que esta foi positivamente correlacionada com o suporte social oferecido ${ }^{41}$. No presente estudo, ao serem questionados sobre fatores que interferem na QV, o apoio/suporte familiar foi uma das categorias de resposta mencionadas pelos pacientes.

Entretanto, cabe ressaltar a possível sobrecarga para os membros da família e uma grande preocupaçáo de pacientes com câncer em estágio avançado, tanto pelo cuidado dispensado pela família, que influencia a percepção de sobrecarga ${ }^{35,40,41}$, quanto pelo ônus financeiro imposto pela doença, o qual, inclusive, foi destacado por uma das participantes desta pesquisa. Os resultados corroboram uma das diretrizes dos CP que defende a família como unidade de cuidado ${ }^{8,32,36}$.

Conforme observado nos depoimentos e na aplicação do questionário POS, a questão socioeconômica é um relevante fator na concepçáo de QV para os pacientes. Nesse sentido, é fundamental que o médico, em conjunto com a equipe de saúde, compreenda e considere as condiçóes de vida de seu paciente no planejamento do tratamento. Como foi possível observar, a condição financeira foi uma das categorias de resposta mencionadas pelos pacientes, sendo uma variável que precisa ser levada em consideração nos atendimentos domiciliares.

Os achados reforçam a influência dos condicionantes sociais do processo saúde-doença, visto que a QV é direta e indiretamente afetada pelo viés socioeconômico, podendo ser uma limitação na manutenção da QV dos pacientes atendidos em CP domiciliares, corroborando os resultados encontrados em outras pesquisas em nível nacional ${ }^{32,34}$.

\section{CONCLUSÃO}

A integralidade constitui elemento central nos CP e tem grande importância na atenção domiciliar. Entretanto, em se tratando da terminalidade da vida, ainda existem grandes desafios a serem enfrentados no sentido de superar a hegemonia do modelo biomédico na prática da medicina. A fim de alcançar esse objetivo, é necessário investir em novos modelos de intervenção que valorizem as diferentes dimensões do cuidado à saúde.

$\mathrm{Na}$ atualidade, com raras exceçôes, as faculdades de medicina náo preparam adequadamente seus acadêmicos a lidarem com o processo de finitude da vida, em que pese o fortalecimento dos $\mathrm{CP}$ no cenário brasileiro. Como consequência, muitos médicos nem sequer conhecem o que de fato significam $\mathrm{CP}$, carecendo de conhecimentos e técnicas de intervenção voltados à minimizaçáo da dor e do sofrimento na assistência integral a tais pacientes.

Os CP incluem a escuta ativa do paciente e de sua família, tendo em vista que há sempre a possibilidade de fazer algo de forma técnica, sublime e amorosa. Dessa maneira, é fundamental que as políticas públicas em $\mathrm{CP}$ continuem avançando, bem como a abordagem dessa 
temática nas escolas médicas. Como foi possível observar, as privaçôes de ordem econômica merecem atenção especial no planejamento das açóes em saúde, em especial quando se considera a realidade dos pacientes atendidos em hospitais públicos da Região Norte.

A despeito de diversos estudos internacionais, envolvendo avaliação da QV de pacientes com câncer, no Brasil ainda não se contabilizam estudos mais abrangentes sobre a QV de pacientes oncológicos em CP domiciliares. Tal realidade resulta em uma limitaçáo no tocante à comparaçáo dos resultados e ao aprofundamento de reflexôes sobre a influência cultural e regional dos achados obtidos com o presente estudo. Entretanto, aliviar a dor e o sofrimento de pacientes com câncer em CP é muito mais do que utilizar conhecimentos técnicos, é se disponibilizar para aprender a dimensão humana do exercício da medicina, ainda que a morte desponte como um horizonte próximo e inexorável.

\section{CONTRIBUIÇÕES}

Vânia Nazaré Maia dos Santos contribuiu substancialmente na concepção do estudo; na obtenção, análise e interpretação dos dados; na redação e revisão crítica. Ana Cristina Vidigal Soeiro participou como orientadora da pesquisa e na redação e revisão crítica. Cristiane Ribeiro Maués contribuiu substancialmente na concepção do estudo; e na obtenção, análise e interpretação dos dados. Todas as autoras aprovaram a versão final a ser publicada.

\section{DECLARAÇÃO DE CONFLITO DE INTERESSES}

Nada a declarar.

\section{FONTES DE FINANCIAMENTO}

Não há.

\section{REFERÊNCIAS}

1. American Cancer Society. Cancer facts \& figures 2017 [Internet]. Atlanta (GA): American Cancer Society; 2017 [cited 2019 july 23]. Available from: https://www.cancer. org/content/dam/cancer-org/research/cancer-facts-andstatistics/annual-cancer-facts-and-figures/2017/cancerfacts-and-figures-2017.pdf

2. Ferlay J, Soerjomataram I, Dikshit R, et al. Cancer incidence and mortality worldwide: sources, methods and major patterns in GLOBOCAN 2012. Int J Cancer. 2015;136(5):E359-86. doi: https://doi.org/10.1002/ ijc. 29210

3. Instituto Nacional de Câncer José Alencar Gomes da Silva. Estimativa 2020: incidência de câncer no Brasil
[Internet]. Rio de Janeiro: INCA; 2019 [acesso 2019 jul 23]. Disponível em: https://www.inca.gov.br/sites/ufu. sti.inca.local/files//media/document//estimativa-2020incidencia-de-cancer-no-brasil.pdf

4. SIM: Sistema de Informação sobre Mortalidade [Internet]. Brasília, DF: Ministério da Saúde. c2008 [acesso 2019 jul 23]. Disponível em: http://www2. datasus.gov.br/DATASUS/index.php?area $=060701$

5. World Health Organization. National cancer control programmes: policies and managerial guidelines [Internet]. 2nd ed. Geneva: WHO; 2002 [cited 2019 july 23]. Available from: https://www.who.int/cancer/ media/en/408.pdf

6. Meneguin S, Matos TDS, Ferreira MLSM. Perception of cancer patients in palliative care about quality of life. Rev Bras Enferm. 2018;71(4):1998-2004. doi: https:// doi.org/10.1590/0034-7167-2017-0360

7. Leite MAC, Nogueira DA, Terra FS. Social and clinical aspects of oncological patients of a chemotherapy service. Rev Rene. 2015;16(1):38-45. doi: https://doi. org/10.15253/2175-6783.2015000100006

8. Arantes ACQ. A morte é um dia que vale a pena viver [Internet]. Rio de Janeiro: Casa da Palavra; 2016 [acesso 2019 jul 23]. Disponível em: http:// img.travessa.com.br/capitulo/CASA_DA_PALAVRA/ MORTE_E_UM_DIA_QUE_VALE_A_PENA_ VIVER_A-9788544104408.pdf

9. Malta R, Rodrigues B, Priolli DG. Paradigma na formação médica: atitudes e conhecimentos de acadêmicos sobre morte e cuidados paliativos. Rev Bras Educ Med. 2018;42(2):34-44. doi: https://doi.org/10.1590/1981$52712015 \mathrm{v} 42 \mathrm{n} 2 \mathrm{rb} 20170011$

10. World Health Organization. Planning and implementing palliative care services: a guide for programme managers [Internet]. Geneva: WHO; 2016 [cited 2019 July 23]. Available from: http://apps.who.int/iris/bitstre am/10665/250584/1/9789241565417-eng.pdf

11. Ministério da Saúde (BR), Gabinete do Ministro, Comissão Intergestores Tripartite. Resolução no 41 , de 31 de outubro de 2018. Dispóe sobre as diretrizes para a organização dos cuidados paliativos, à luz dos cuidados continuados integrados, no âmbito Sistema Único de Saúde (SUS) [Internet]. Diário Oficial da União. 2018 nov 23; Seção 1:276 [acesso 2019 jul 23]. Disponível em: http://portalarquivos2.saude.gov.br/images/pdf/2018/ novembro/23/RESOLUCAO-N41.pdf

12. Faller JW, Brusnicki PH, Zilly A, et al. Perfil de idosos acometidos por câncer em cuidados paliativos em domićlilio. Rev Kairós. 2016;19(Esp 22):29-43. doi: https://doi. org/10.23925/2176-901X.2016v19iEspecial22p29-43

13. Simão VM, Mioto RCT. O cuidado paliativo e domiciliar em países da América Latina. Saúde Deb. 2016;40(108):156-69. doi: https://doi. org/10.1590/0103-1104-20161080013 
14. MacArtney JI, Broom A, Kirby E, et al. Locating care at the end of life: burden, vulnerability, and the practical accomplishment of dying. Sociol Health Iln. 2016;38(3):479-92. doi: https://doi.org/10.1111/14679566.12375

15. Jorge R, Calanzani N, Freitas A, et al. Preferência por morrer em casa e fatores associados de pessoas idosas da cidade de Belo Horizonte, Brasil. Ciênc Saúde Coletiva. 2019;24(8):3001-12. doi: https://doi.org/10.1590/141381232018248.24102017

16. Danielsen BV, Sand AM, Rosland JH, et al. Experiences and challenges of home care nurses and general practitioners in home based palliative care: a qualitative study. BMC Palliative Care. 2018;17(1):95. doi: https:// doi.org/10.1186/s12904-018-0350-0

17. Bone AE, Gao W, Gomes B, et al. Factors associated with transition from community settings to hospital as place of death for adults aged 75 and older: a population based mortality follow back survey. J Am Geriatr Soc. 2016;64(11):2210-17. doi: https://doi.org/10.1111/ jgs. 14442

18. Catania G, Beccaro M, Costantini M, et al. What are the components of interventions focused on quality-of-life assessment in palliative care practice? A systematic review. J Hosp Palliative Nurs. 2016;18(4):310-6. doi: https:// doi.org/10.1097/NJH.0000000000000248

19. Ferreira MLL, Souza AI, Ferreira LOC. Qualidade de vida relacionada à saúde de idosos em tratamento quimioterápico. Rev Bras Geriatr Gerontol. 2015;18(1):165-77. doi: https://doi.org/10.1590/18099823.2015.14008

20. Kagawa-Singer M, Padilla GV, Ashing-Giwa K. Healthrelated quality of life and culture. Semin Oncol Nurs. 2010;26(1):59-67. doi: https://doi.org/10.1016/j. soncn.2009.11.008

21. A better way to care for the dying. The Economist Newspaper [Internet]. 2017 Apr 29 [cited 2019 July 23]; End-of-life care. Available from: https://www. economist.com/international/2017/04/29/a-better-wayto-care-for-the-dying

22. McCaffrey N, Bradley S, Ratcliffe J, et al. What aspects of quality of life are important from palliative care patients' perspectives? A systematic review of qualitative research. J Pain Symptom Manage. 2016;52(2):318-328.e5. doi: https://doi. org/10.1016/j.jpainsymman.2016.02.012

23. Sawada NO, Nicolussi AC, Paula JM, et al. Quality of life of Brazilian and Spanish cancer patients undergoing chemotherapy: an integrative literature review. Rev Latino-Am Enfermagem. 2016;24:e2688. doi: https:// doi.org/10.1590/1518-8345.0564.2688

24. Correia DS, Bezerra MES, Lucena TS, et al. Cuidados paliativos: importância do tema para discentes de graduação em medicina. Rev Bras Educ Med.
2018;42(3):78 86. doi: https://doi.org/10.1590/1981$52712015 \mathrm{v} 42 \mathrm{n} 3 \mathrm{rb} 20170105 . \mathrm{r} 1$

25. Bastos BR, Pereira AKS, Castro CC, et al. Perfil sociodemográfico dos pacientes em cuidados paliativos em um hospital de referência em oncologia do estado do Pará, Brasil. Rev Pan-Amaz Saude. 2018;9(2):31-36. doi: https://doi.org/10.5123/s2176-62232018000200004

26. Nicolussi AC, Sawada NO, Cardozo FMC, et al. Qualidade de vida relacionada à saúde de pacientes com câncer em quimioterapia. Rev Rene [Internet]. 2014 [2019 jul 23];15(1):132-40. Disponível em: https:// www.researchgate.net/profile/Adriana_Nicolussi2/ publication/282978806_qualidade_de_vida_ relacionada_a_saude_de_pacientes_com_cancer_em_ quimioterapia/links/5625251108aeedae57dae334.pdf

27. Antunes YPPV, Bugano DDG, Giglio A, et al. Características clínicas e de sobrevida global em pacientes oncológicos idosos num centro oncológico terciário. Einstein. 2015;13(4):487-91. doi: http://doi. org/10.1590/S1679-45082015AO3067

28. Instituto Brasileiro de Geografia e Estatística [Internet]. Rio de Janeiro: IBGE; c2020. Censo 2010; 2010 [acesso 2016 nov 6]. Disponível em: http://www.censo2010. ibge.gov.br/

29. Sociedade Brasileira de Cancerologia [Internet]. Salvador: Sociedade Brasileira de Cancerologia; c2020. Câncer ginecológico; 2016 out 5 [acesso 2016 nov 9]. Disponível em: http://www.sbcancer.org.br/cancer-ginecologico/

30. Damacena GN, Szwarcwald CL, Malta DC, et al. O processo de desenvolvimento da pesquisa nacional de saúde no Brasil, 2013. Epidemiol Serv Saúde. 2015;24(2):197-206. doi: https://doi.org/10.5123/ S1679-49742015000200002

31. Silva MJS, Bergmann A, Siqueira ASE, et al. Influência das iniquidades sociais e dos cuidados de saúde na incidência e mortalidade por câncer. Rev Bras Canc. 2018;64(4):459-60. doi: https://doi.org/10.32635/21769745.RBC.2018v64n4.211

32. Prager GW, Braga S, Bystricky B, et al. Global cancer control: responding to the growing burden, rising costs and inequalities in access. ESMO Open. 2018;3(2):e000285. doi: https://doi.org/10.1136/ esmoopen-2017-000285

33. Noce CW, Rebelo MS. Avaliação da relação entre tamanho do tumor e características sociais em pacientes com carcinoma de células escamosas bucal. Rev Bras Cancerol [Internet]. 2008 [acesso 2019 jul 23]; 54(2):123-9. Disponível em: http://www1.inca.gov.br/ rbc/n_54/v02/pdf/artigo_2_pag_123a129.pdf

34. Schneider IJC, D'Orsi E. Sobrevida em cinco anos e fatores prognósticos em mulheres com câncer de mama em Santa Catarina, Brasil. Cad Saúde Pública. 2009;25(6):1285-96. doi: https://doi.org/10.1590/ S0102-311X2009000600011 
35. Dugno MLG, Soldatelli JS, Daltoé T, et al. Perfil do câncer de mama e relação entre fatores de risco e estadiamento clínico em hospital do Sul do Brasil. Rev Bras Oncol Clin [Internet]. 2014 [acesso 2019 jul 23];10(36):60-6. Disponível em: https://www.sboc.org. br/sboc-site/revista-sboc/pdfs/36/artigo3.pdf.

36. Rushton L, Bagga S, Bevan R, et al. Occupation and cancer in Britain. Br J Cancer. 2010;102(9):1428-37. doi: https://doi.org/10.1038/sj.bjc.6605637

37. Queiroz L, Sousa F, Marques H. Influência dos factores socio-demográficos e clínicos na qualidade de vida relacionada com a saúde dos doentes hematooncológicos. Arq Med [Internet]. 2010 [acesso 2019 jul 23];24(3):79-87. Disponível em: http://www.scielo. mec.pt/scielo.php?script $=$ sci_arttext $\&$ pid $=$ S0871 34132010000300001\&lng=pt

38. Mesquita AC, Chaves ECL, Avelino CCV, et al. A utilização do enfrentamento religioso/espiritual por pacientes com câncer em tratamento quimioterápico. Rev Latino-Am Enfermagem. 2013;21(2):24-29. doi: https://doi.org/10.1590/S0104-11692013000200010

39. Facina T. Diretrizes para a detecção precoce do câncer de mama no Brasil. Rev Bras Cancerol. 2016;62(1):5960. doi: https://doi.org/10.32635/2176-9745. RBC.2016v62n1.415

40. Minson FP, Garcia JBS, Oliveira Júnior JO, et al., editores. II Consenso Nacional de Dor Oncológica [Internet]. São Paulo: Moreira Jr. Editora; 2011 [acesso 2019 jul 23]. Disponível em: http://docplayer.com. br/50484685-Ii-consenso-nacional-de-dor-oncologica. html

41. Yang L, Song WP, Chen ZL, et al. [Correlation between social support and quality of life in patients with breast cancer at different periods of treatment]. Zhonghua Zhong Liu Za Zhi. 2017;39(3):202-6. doi: https:// doi.org/10.3760/cma.j.issn.0253-3766.2017.03.009 Chinese. 